\title{
Time-like Ruled Surface in One-Parameter Hyperbolic Dual Spherical Motions
}

\author{
Monia F. Naghi, ${ }^{1}$ Rashad A. Abdel-Baky $\mathbb{D}^{2},{ }^{2}$ and Fatemah Mofarreh ${ }^{3}{ }^{3}$ \\ ${ }^{1}$ Department of Mathematics, Faculty of Sciences, King Abdulaziz University, Saudi Arabia \\ ${ }^{2}$ Department of Mathematics, Faculty of Sciences, University of Assiut, 71516 Assiut, Egypt \\ ${ }^{3}$ Mathematical Science Department, Faculty of Sciences, Princess Nourah Bint Abdulrahman, Riyadh 11546, Saudi Arabia
}

Correspondence should be addressed to Fatemah Mofarreh; fyalmofarrah@pnu.edu.sa

Received 16 October 2021; Accepted 6 December 2021; Published 17 January 2022

Academic Editor: Gueo Grantcharov

Copyright (C) 2022 Monia F. Naghi et al. This is an open access article distributed under the Creative Commons Attribution License, which permits unrestricted use, distribution, and reproduction in any medium, provided the original work is properly cited.

\begin{abstract}
In this work, we introduce a time-like ruled surface in one-parameter hyperbolic dual spherical motions. This provides the ability to derive some formulae of surface theory into line spaces. Then, a time-like Plücker conoid associated with the motion has been obtained, and its kinematic geometry is researched in detail. Consequently, a characterization for a time-like ruled surface to be a constant Disteli-axis is derived and investigated in detail. At last, we have discussed some special cases which lead to some special time-like ruled surfaces such as the time-like helicoids, Lorentzian sphere, and time-like cone.
\end{abstract}

\section{Introduction}

In spatial kinematics, the movement of an oriented line over a curve performs ruled surface. These oriented lines are said to be generators (rulings), and each curve that intersects all the generators is called a directrix (base curve) $[1-3]$. One of the most suitable methods to study the movement of oriented line space seems to create a relationship between this space, dual numbers, and dual vector calculus. Dual numbers were first introduced by W. Clifford; after him, E. Study used it as an instrument for his research on line geometry and kinematics. He devoted special care to the impersonation of oriented lines by dual unit vectors and defined the mapping that is known by his name. The E. Study map states that "The set of all oriented lines in Euclidean 3-space $\mathbb{E}^{3}$ is directly linked to the set of points on the dual unit sphere in the dual 3-space $\mathbb{D}^{3}$." Thus, the differential geometry of ruled surfaces based on the E. Study map has rederived the curvature theory of a line trajectory and exposed the fundamental curvature functions that describe the shape of ruled surface (see, for example, [4-11]).

In the Minkowski 3-space $\mathbb{E}_{1}^{3}$, the investigation of ruled surfaces is more motivating than the Euclidean case, since the Euclidean metric can only be positive whereas the Lorentzian metric can be negative, positive, or zero. Therefore, the kinematics and geometric explanations can be widely different. Hence, if we replace the Minkowski 3-space $\mathbb{E}_{1}^{3}$ as an alternative of the Euclidean 3 -space $\mathbb{E}^{3}$, the E. Study map can be given as follows: "the time-like (resp., space-like) oriented lines are represented with the time-like (resp., space-like) dual points on the hyperbolic (resp., Lorentzian) dual unit sphere in the Lorentzian dual 3-space $\mathbb{D}_{1}^{3}[10,11]$." It means that a regular curve on $\mathrm{H}_{+}^{2}$ represents a time-like ruled surface at $\mathbb{E}_{1}^{3}$. Similarly, the space-like (resp., time-like) curve on $\mathbb{S}_{1}^{2}$ represents a time-like (resp., space-like) ruled surface at $\mathbb{E}_{1}^{3}$. In view of its relationships with engineering and physical sciences in Minkowski space, many geometers and engineers have studied and gained many ownerships of the ruled surfaces (see [8-13]).

However, to the best of the authors' knowledge, no literature exists regarding the time-like ruled surface with constant Disteli-axis from one-parameter hyperbolic dual spherical motions. Thus, the present study hopes to serve such a need. This work offers an approach for constructing time-like ruled surfaces with constant Disteli-axis by using E. Study map. Hence, the well-known formulae of Hamilton, 
Mannheim, and Dupin's indicatrix of surfaces theory are proved for the line space. Moreover, a time-like Plücker conoid associated with the motion has been derived and it is shown that the principal axes of it are located at its center. Then, some characterization equations for special time-like ruled surfaces such as the time-like helicoid, the Lorentzian sphere, and the time-like cone are obtained and investigated.

\section{Basic Concepts}

We start with basic concepts on the Minkowski 3-space $\mathbb{E}_{1}^{3}$, the theory of dual numbers, dual Lorentzian vectors, and E. Study map, for example, [1-3, 14-17]. A dual number $A$ is a number $a+\varepsilon a^{*}$, where $a, a^{*}$ in $\mathbb{R}$ and $\varepsilon$ is a dual unit with the property that $\varepsilon^{2}=0$. Then, the set

$$
\mathbb{D}^{3}=\left\{A:=\mathbf{a}+\varepsilon \mathbf{a}^{*}=\left(A_{1}, A_{2}, A_{3}\right)\right\},
$$

together with the Lorentzian scalar product

$$
<\mathbf{A}, \mathbf{A}>=-A_{1}^{2}+A_{2}^{2}+A_{3}^{2}
$$

forms the so-called dual Lorentzian 3-space $\mathbb{D}_{1}^{3}$. Thus, a point $A=\left(A_{1}, A_{2}, A_{3}\right)^{t}$ has dual coordinates $A_{i}=\left(a_{i}+\varepsilon a_{i}^{*}\right)$ $\in \mathbb{D}$. If $\mathbf{A}$ is space-like or time-like dual vector the norm $\|\mathbf{A}\|$ of $\mathbf{A}$ is defined by

$$
\begin{gathered}
\|\mathbf{A}\|=\sqrt{|<\mathbf{A}, \mathbf{A}\rangle \mid}=\sqrt{|<\mathbf{a}, \mathbf{a}\rangle \mid}+\varepsilon \frac{1}{2 \sqrt{|<\mathbf{a}, \mathbf{a}\rangle \mid}} \frac{<\mathbf{a}, \mathbf{a}\rangle}{|<\mathbf{a}, \mathbf{a}\rangle \mid} \\
\cdot 2\left\langle\mathbf{a}, \mathbf{a}^{*}\right\rangle=\|\mathbf{a}\|+\varepsilon \frac{1}{\|\mathbf{a}\|} \frac{<\mathbf{a}, \mathbf{a}\rangle}{|<\mathbf{a}, \mathbf{a}\rangle \mid}\left\langle\mathbf{a}, \mathbf{a}^{*}\right\rangle
\end{gathered}
$$

If $\mathbf{a}$ is space-like, we have

$$
\|\mathbf{A}\|=\|\mathbf{a}\|+\varepsilon \frac{1}{\|\mathbf{a}\|}<\mathbf{a}, \mathbf{a}^{*}>=\|\mathbf{a}\|\left(1+\varepsilon \frac{1}{\|\mathbf{a}\|^{2}}<\mathbf{a}, \mathbf{a}^{*}>\right) .
$$

If $\mathbf{a}$ is time-like, we have

$$
\|\mathbf{A}\|=\|\mathbf{a}\|-\varepsilon \frac{1}{\|\mathbf{a}\|}<\mathbf{a}, \mathbf{a}^{*}>=\|\mathbf{a}\|\left(1-\varepsilon \frac{1}{\|\mathbf{a}\|^{2}}<\mathbf{a}, \mathbf{a}^{*}>\right) .
$$

Therefore, $\mathbf{A}$ is called a space-like (resp., time-like) dual unit vector if $\langle\mathbf{A}, \mathbf{A}\rangle=1$ (resp. $\langle\mathbf{A}, \mathbf{A}\rangle=-1$ ). The hyperbolic and Lorentzian dual unit spheres, respectively, are

$$
\begin{aligned}
& \mathbb{H}_{+}^{2}=\left\{\mathbf{A} \in \mathbb{D}_{1}^{3} \mid-A_{1}^{2}+A_{2}^{2}+A_{3}^{2}=-1\right\}, \\
& \mathbb{S}_{1}^{2}=\left\{\mathbf{A} \in \mathbb{D}_{1}^{3} \mid-A_{1}^{2}+A_{2}^{2}+A_{3}^{2}=1\right\} .
\end{aligned}
$$

Theorem 1. There is a one-to-one correspondence between space-like (resp., time-like) oriented lines in the Minkowski 3 -space $\mathbb{E}_{1}^{3}$ and ordered pairs of vectors $\left(\mathbf{a}, \mathbf{a}^{*}\right)$ such that

$$
\|\mathbf{A}\|^{2}= \pm 1 \Leftrightarrow\|\mathbf{a}\|^{2}= \pm 1, \quad<\mathbf{a}, \mathbf{a}^{*}>=0
$$

where $a_{i}, a_{i}^{*}(i=1,2,3)$ of $\mathbf{a}$ and $\mathbf{a}^{*}$ are called the normed Plücker coordinates of the line.

According to Theorem 1, four independent parameters locate a line complex, so it is reasonable to intersect any two of line complexes and gain a definite number of lines (line congruence) with common properties. The intersection of two independent linear congruences carries out a differentiable family of straight lines (a ruled surface). Ruled surfaces (such as cylinders and cones) include rulings where the tangent plane relates the surface over the entire line (torsal lines) [1-3]. Also, we have the E. Study's map: the dual unit spheres are shaped as a pair of conjugate hyperboloids. The ring-shaped hyperboloid correlates with the set of space-like lines, the common asymptotic cone correlates the set of null (light-like) lines, and the oval-shaped hyperboloid forms the set of time-like lines, and opposite points of each hyperboloid perform the pair of obverse vectors on a line (see Figure 1). Therefore, a regular curve on $\mathbb{H}_{+}^{2}$ represents a time-like ruled surface in $\mathbb{E}_{1}^{3}$. Similarly, a regular curve on $\mathbb{S}_{1}^{2}$ represents a space-like or time-like ruled surface in $\mathbb{E}_{1}^{3}$.

2.1. One-Parameter Hyperbolic Dual Spherical Motions. Consider two hyperbolic dual unit spheres $\mathrm{H}_{+m}^{2}$ and $\mathrm{H}_{+f}^{2}$. Suppose that $\mathbf{O}$ is the common center, and two orthonormal dual frames $\left\{\mathbf{O} ; \mathbf{R}_{1}\right.$ (time - like $\left.), \mathbf{R}_{2}, \mathbf{R}_{3}\right\}$, and $\left\{\mathbf{O} ; \mathbf{F}_{1}(\right.$ time - like), $\left.\mathbf{F}_{2}, \mathbf{F}_{3}\right\}$ be rigidly linked to $\mathbb{H}_{+m}^{2}$ and $\mathbb{H}_{+f}^{2}$, respectively. If we suppose that $\left\{\mathbf{O} ; \mathbf{F}_{1}, \mathbf{F}_{2}, \mathbf{F}_{3}\right\}$ is fixed, whereas the elements of the set $\left\{\mathbf{O} ; \mathbf{R}_{1}, \mathbf{R}_{2}, \mathbf{R}_{3}\right\}$ are functions of a real parameter $t \in \mathbb{R}$ (say the time), then we say that $\mathrm{H}_{+m}^{2}$ moves with respect to $\mathrm{H}_{+f}^{2}$. This motion is called a one-parameter hyperbolic dual spherical motions and will denoted by $\mathrm{H}_{+m}^{2} / \mathrm{H}_{+f}^{2}$. If the dual unit spheres $\mathrm{H}_{+m}^{2}$ and $\mathrm{H}_{+f}^{2}$ correspond to the line spaces $\mathbb{L}_{m}$ and $\mathbb{L}_{f}$, respectively, then $\mathbb{H}_{+m}^{2} / \mathbb{H}_{+f}^{2}$ represents the one-parameter Lorentzian spatial motion $\mathbb{L}_{m} / \mathbb{L}_{f}$. Therefore, $\mathbb{L}_{m}$ is the moving Lorentzian space with respect to the fixed Lorentzian space $\mathbb{L}_{f}$ in $\mathbb{E}_{1}^{3}$. By putting $\left.<\mathrm{F}_{i}, R_{j}\right\rangle=L_{i j}$ and introducing the dual matrix $L=\left(L_{i j}\right)$, we can write the E. Study map in the matrix form as follows:

$$
\mathbb{H}_{+m}^{2} / \mathbb{H}_{+f}^{2}:\left(\begin{array}{c}
\mathbf{F}_{1} \\
\mathbf{F}_{2} \\
\mathbf{F}_{3}
\end{array}\right)=\left(\begin{array}{ccc}
L_{11} & L_{12} & L_{13} \\
L_{21} & L_{22} & L_{23} \\
L_{31} & L_{32} & L_{33}
\end{array}\right)\left(\begin{array}{l}
\mathbf{R}_{1} \\
\mathbf{R}_{2} \\
\mathbf{R}_{3}
\end{array}\right) .
$$

It then follows that the signature matrix $\epsilon$, describing the scalar product in $\mathbb{D}_{1}^{3}$, is given by [15]

$$
\epsilon=\left(\begin{array}{ccc}
-1 & 0 & 0 \\
0 & 1 & 0 \\
0 & 0 & 1
\end{array}\right)
$$




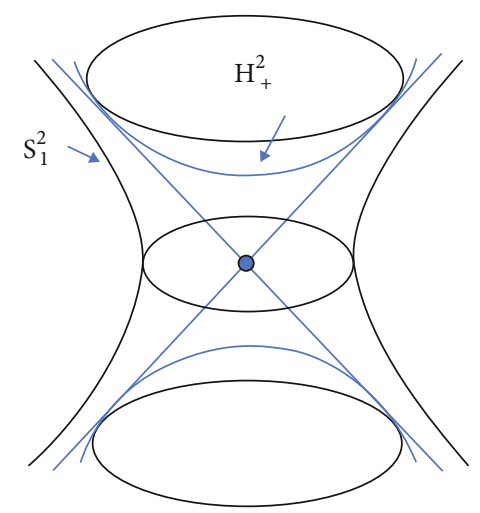

FIgure 1: The hyperbolic and Lorentzian dual unit spheres.

The dual matrix $L:=\left(L_{i j}\right)=\left(l_{i j}\right)+\varepsilon\left(l_{i j}^{*}\right)$ has the property that $L^{T}=\epsilon L^{-1} \epsilon, L^{-1}=L^{T} \epsilon L$. Hence, we have

$$
L L^{-1}=L \epsilon L^{T} \epsilon=L^{-1} L=\epsilon L^{T} \epsilon L=I,
$$

where $I$ is the $3 \times 3$ unit matrix. This result indicates that when a one-parameter Lorentzian spatial motion is given in $\mathbb{E}_{1}^{3}$, we can find a corresponding dual Lorentzian orthogonal $3 \times 3$ matrix $L(t)=\left(L_{i j}\right)$, where $\left(L_{i j}\right)$ are dual functions of one variable $t \in \mathbb{R}$. As the set of real Lorentzian orthogonal matrices, the set of Lorentzian dual orthogonal $3 \times 3$ matrices, denoted by $\mathbb{O}\left(\mathbb{D}_{1}^{3 \times 3}\right)$, forms a group with matrix multiplication as the group operation (real Lorentzian orthogonal matrices are a subgroup of Lorentzian dual orthogonal matrices). The identity element of $\mathbb{O}\left(\mathbb{D}_{1}^{3 \times 3}\right)$ is the $3 \times 3$ unit matrix. Since the center of the hyperbolic dual unit sphere in $\mathbb{D}_{1}^{3}$ must remain fixed, the transformation group in $\mathbb{D}_{1}^{3}$ (the image of Lorentzian motions in the Minkowski 3-space $\mathbb{E}_{1}^{3}$ ) does not contain any translations.

The Lie algebra $\mathbb{L}\left(\mathbb{O}_{\mathbb{D}_{1}^{3 \times 3}}\right)$ of the group $\mathbb{G} \mathbb{L}$ of $3 \times 3$ positive orthogonal dual matrices $L$ is the algebra of skewadjoint $3 \times 3$ dual matrices

$$
\Omega(t):=L^{\prime} \epsilon L^{T} \epsilon=\left(\begin{array}{ccc}
0 & \Omega_{3} & -\Omega_{2} \\
\Omega_{3} & 0 & \Omega_{1} \\
\Omega_{2} & -\Omega_{1} & 0
\end{array}\right)=\left(\begin{array}{c}
-\Omega_{1} \\
\Omega_{2} \\
\Omega_{3}
\end{array}\right),
$$

where dash indicates the differential with respect to the real parameter $t \in \mathbb{R}$. Then, the derivative equation of $\mathbb{H}_{+m}^{2} / \mathbb{H}_{+f}^{2}$ is

$$
\left(\begin{array}{l}
\mathbf{R}_{1}^{\prime} \\
\mathbf{R}_{2}^{\prime} \\
\mathbf{R}_{3}^{\prime}
\end{array}\right)=\left(\begin{array}{ccc}
0 & \Omega_{3} & -\Omega_{2} \\
\Omega_{3} & 0 & \Omega_{1} \\
\Omega_{2} & -\Omega_{1} & 0
\end{array}\right)\left(\begin{array}{l}
\mathbf{R}_{1} \\
\mathbf{R}_{2} \\
\mathbf{R}_{3}
\end{array}\right)=\Omega \times\left(\begin{array}{l}
\mathbf{R}_{1} \\
\mathbf{R}_{2} \\
\mathbf{R}_{3}
\end{array}\right) .
$$

$\Omega(t)=\omega+\varepsilon \omega^{*}=\left(\Omega_{1}, \Omega_{2}, \Omega_{3}\right)$ is called the instantaneous dual rotation vector of $\mathbb{H}_{+m}^{2} / \mathbb{H}_{+f}^{2}$. The real part $\omega$ and dual part $\omega^{*}$, respectively, correspond to the instantaneous rotational differential velocity vector and the instantaneous translational differential velocity vector of the motion $\mathbb{L}_{m} / \mathbb{L}_{f}$.

\section{Main Results}

During the motion $\mathbb{L}_{m} / \mathbb{L}_{f}$, any fixed time-like line $\mathbf{X} \in \mathbb{L}_{m}$ traces a time-like ruled surface in $\mathbb{L}_{f}$ which will be denoted by $(X)$. In spatial kinematics, such time-like ruled surface is called time-like line trajectory. In order study the geometrical properties of $(X)$, we choose a moving frame, called the Blaschke frame, associated with point on $\mathbb{H}_{+m}^{2}$ as

$\mathbf{X}=\mathbf{X}(t), \mathbf{T}(t):=\mathbf{t}(t)+\varepsilon \mathbf{t}^{*}(t)=\frac{\mathbf{X}^{\prime}}{\left\|\mathbf{X}^{\prime}\right\|}, \mathbf{G}(t):=\mathbf{g}(t)+\varepsilon \mathbf{g}^{*}(t)=\mathbf{X} \times \mathbf{T}$.

Then, we find

$$
\left.\begin{array}{l}
-<\mathbf{X}, \mathbf{X}>=<\mathbf{T}, \mathbf{T}>=<\mathbf{G}, \mathbf{G}>=1, \\
<\mathbf{X}, \mathbf{T}>=<\mathbf{X}, \mathbf{G}>=<\mathbf{G}, \mathbf{T}>=0, \\
\mathbf{G} \times \mathbf{X}=\mathbf{T}, \mathbf{T} \times \mathbf{G}=-\mathbf{X} .
\end{array}\right\}
$$

$\mathbf{X}, \mathbf{T}$, and $\mathbf{G}$ are three dual unit vectors corresponding to three concurrent mutually perpendicular lines $\mathbb{E}_{1}^{3}$, and they intersect at a point $\mathbf{C}$ on $\mathbf{X}$ called the central point. $\mathbf{G}$ is the limit position of the common perpendicular to $\mathbf{X}(t)$ and $\mathbf{X}(t+d t)$ and is called the central tangent of $(X)$ at the central point. The line $T$ is called the central normal of $(X)$ at the central point. Thus, the motion $\mathbb{H}_{+m}^{2} / \mathbb{H}_{+f}^{2}$ is given by [9-11]

$$
\mathbb{H}_{+m}^{2} / \mathbb{H}_{+f}^{2}:\left(\begin{array}{c}
\mathbf{X}^{\prime} \\
\mathbf{T}^{\prime} \\
\mathbf{G}^{\prime}
\end{array}\right)=\left(\begin{array}{ccc}
0 & P & 0 \\
P & 0 & Q \\
0 & -Q & 0
\end{array}\right)\left(\begin{array}{c}
\mathbf{X} \\
\mathbf{T} \\
\mathbf{G}
\end{array}\right)=\boldsymbol{\Omega} \times\left(\begin{array}{c}
\mathbf{X} \\
\mathbf{T} \\
\mathbf{G}
\end{array}\right),
$$

where

$$
\begin{aligned}
P(t) & =p(t)+\varepsilon p^{*}(t)=\left\|\mathbf{X}^{\prime}\right\|, Q(t):=q(t)+\varepsilon q^{*}(t) \\
& =\operatorname{det}\left(\mathbf{X}, \mathbf{X}^{\prime}, \mathbf{X}^{\prime \prime}\right)\left\|\mathbf{X}^{\prime}\right\|^{-2},
\end{aligned}
$$

are called Blaschke invariants of $(X)$. The tangent of the striction curve may be written as

$$
\mathbf{C}^{\prime}=-q^{*} \mathbf{x}+p^{*} \mathbf{g} .
$$

The invariants $p(t), p^{*}(t), q(t)$, and $q^{*}(t)$ are called the structure functions of the ruled surface $(X)$. The distribution parameters of the ruled surfaces $(X),(T)$, and $(G)$, respectively, are 


$$
\mu(t)=\frac{p^{*}}{p}, \delta(t)=\frac{p p^{*}+q q^{*}}{q^{2}+p^{2}}, \Gamma(t)=\frac{q^{*}}{q} .
$$

Definition 2. A nondevelopable ruled surface is defined as a constant parameter ruled surface if the structure functions $\mu(t), \delta(t)$, and $\Gamma(t)$ are all constant.

Now, under the hypothesis that $|Q|>|P|$, we have the evolute of $\mathbf{X} \in \mathbb{H}_{+}^{2}$ as

$$
\mathbf{B}(t)=\mathbf{b}(t)+\varepsilon \mathbf{b}^{*}(t)=\frac{\boldsymbol{\Omega}}{\|\boldsymbol{\Omega}\|}=\frac{Q \mathbf{X}+P \mathbf{G}}{\sqrt{Q^{2}-P^{2}}} .
$$

It is clear that $\mathbf{B}$ is the Disteli-axis (curvature-axis or striction-axis) of $(X)$. Let $\Psi=\psi+\varepsilon \psi^{*} \geq 0$ stand for the radius of curvature between $\mathbf{X}$ and $\mathbf{B}$. Then, we can write

$$
\mathbf{B}(t)=\cosh \Psi \mathbf{X}+\sinh \Psi \mathbf{G}, \text { with } \operatorname{coth} \Psi=\frac{Q}{P} .
$$

One of the invariants of the dual curve $\mathbf{X} \in \mathbb{H}_{+}^{2}$ is

$$
\Sigma(t):=\gamma(t)+\varepsilon(\Gamma(t)-\mu(t) \gamma(t))=\frac{Q}{P},
$$

called the dual geodesic curvature. Here, $\gamma(t)=q / p$ is the geodesic curvature of the hyperbolic spherical image curve $t \in I \mapsto \mathbf{x}(t)$ of $(X)$. The trigonometric hyperbolic function $\Psi$ can be written as

$$
\operatorname{coth} \Psi=\operatorname{coth} \psi-\varepsilon \psi^{*} \frac{1}{\sinh ^{2} \psi}
$$

Thus, from the real and dual parts of Equations (21) and (22), respectively, we find

$$
\begin{gathered}
\gamma(t)=\operatorname{coth} \psi=\frac{q}{p}, \\
\psi^{*}(t)=\frac{1}{2}(\mu-\Gamma) \sinh 2 \psi .
\end{gathered}
$$

$\psi^{*}$ is the normal distance along $\mathbf{T}$ measured from $\mathbf{B}$ to $\mathbf{X}$.

3.1. Kinematic-Geometry and Time-like Plücker Conoid. We now are interested in researching the kinematic geometry of $(X)$. Therefore, we are going to make a detailed study of the Blaschke invariants $P(t)$ and $Q(t)$. To carry this out, from Equation (15), we can write the following equations:

$\mathbf{X}^{\prime}(t)=(\|\Omega\| \mathbf{B}) \times \mathbf{X}, \mathbf{T}^{\prime}(t)=(\|\Omega\| \mathbf{B}) \times \mathbf{T}, \mathbf{G}^{\prime}(t)=(\|\Omega\| \mathbf{B}) \times \mathbf{G}$

Hence, at any instant, it is seen that $\|\Omega\|=\Omega=\omega+\varepsilon \omega^{*}$ is the dual angular speed of the motion $\mathbb{H}_{+m}^{2} / \mathbb{H}_{+f}^{2}$ about the Disteli-axis B. Thus,

$$
\omega(t)=\sqrt{q^{2}-p^{2}}, \omega^{*}(t)=\frac{q q^{*}-p p^{*}}{\sqrt{q^{2}-p^{2}}}, \text { with }|q|>|p| \text {, }
$$

corresponding to the rotational angular speed and translational angular speed of the motion $\mathbb{L}_{m} / \mathbb{L}_{f}$ along $\mathbf{B}$, respectively.

Hence, the next corollary can be given:

Corollary 3. During the motion $\mathbb{L}_{m} / \mathbb{L}_{f}$, at any instant $t$, the pitch can be given by

$$
h(t):=\frac{<\omega, \omega^{*}>}{\|\omega\|^{2}}=\Gamma \cosh ^{2} \psi-\mu \sinh ^{2} \psi .
$$

It is clear that if the dual vector $\Omega=\omega+\varepsilon \omega^{*}$ is given, then the following can be specified:

(i) The time-like Disteli-axis $\mathbf{B}$ is specified by Equation (20)

(ii) The dual angular speed is $\|\Omega\|=\omega(1+\varepsilon h)$

(iii) If $\mathbf{y}$ is a point on the time-like Disteli-axis $\mathbf{B}$, then

$$
\mathbf{y}(t, v)=\mathbf{b} \times \mathbf{b}^{*}+v \mathbf{b}, v \in \mathbb{R},
$$

is a nondevelopable time-like ruled surface $(B)$

(iv) If the motion $\mathbb{L}_{m} / \mathbb{L}_{f}$ is pure rotation, that is, $h(u)$ $=0$, then

$$
\mathbf{B}(t)=\mathbf{b}(t)+\varepsilon \mathbf{b}^{*}(t)=\frac{1}{\|\omega\|}\left(\omega+\varepsilon \omega^{*}\right)
$$

It is interesting to note that if $h(t)=0$, and $\|\omega\|^{2}=-1$, then $\Omega$ is a time-like oriented line. However, in the case when the motion is pure translational, i.e., $\Omega=0+\varepsilon \omega^{*}$, we set $\omega^{*}=\left\|\Omega^{*}\right\|, \omega^{*} \mathbf{b}=\omega^{*}$ and select an arbitrary $\mathbf{b}^{*}$ under $\omega^{*}$ $\neq 0$; otherwise, the time-like unit vector $\mathbf{b}$ can be chosen arbitrarily, too.

The Equations in (24) and (27), respectively, are Minkowski versions of the Mannheim and Hamilton formulae of surface theory in Euclidean 3-space. Now, we give geometric interpretations of these formulae. Next, the parametric representation of $\psi^{*}$ is time-like Plücker conoid or cylindroid as follows: consider $\mathbf{T}$ is coincident with the space-like $y$-axis of a fixed Minkowski frame (oxyz), while the position of the time-like dual unit vector $\mathbf{B}$ is given by angle $\psi$ and distance $\psi^{*}$ on the space-like positive $y$-axis. The time-like dual unit vector $\mathbf{X}$ and the space-like dual unit vector $\mathbf{G}$ can be selected in sense of $x$ - and $z$-axes, respectively. This shows that the dual unit vectors $\mathbf{X}$ and $\mathbf{G}$ together with $\mathbf{T}$ form the coordinate system, as shown in Figure 2. If $\mathbf{y}$ is a point on this time-like Plücker conoid, then we have

$$
M: \mathbf{y}(t, v)=\left(0, \psi^{*}, 0\right)+v(\cosh \psi, 0, \sinh \psi), \quad v \in \mathbb{R} .
$$

Using such representation, the time-like dual unit vectors $\mathbf{B}$ are obviously visible crossing through the $y$-axis. It is easily seen from the last equation and (24) that 


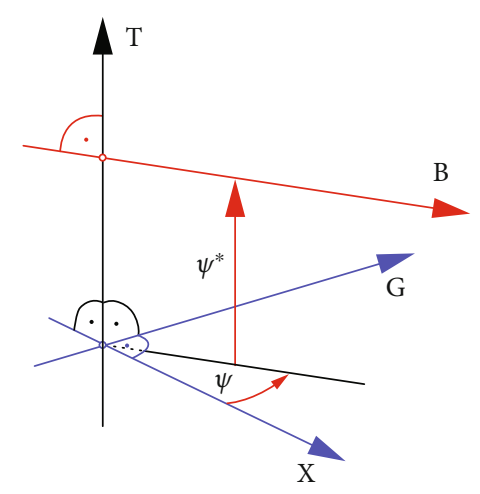

Figure 2: $\mathbf{B}=\cosh \Psi \mathbf{X}+\sinh \Psi \mathbf{G}$.

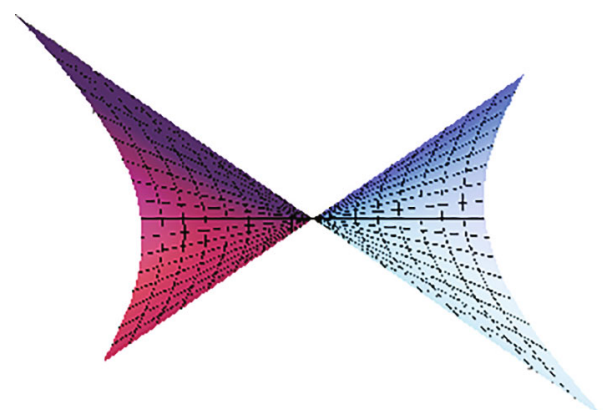

Figure 3: Time-like Plücker conoid.

$\psi^{*}:=y=\frac{1}{2}(\mu-\Gamma) \sinh 2 \psi, x=v \cosh \psi, z=v \sinh \psi$

Here, $\psi^{*}$ gives us the intersection point of the oriented lines $\mathbf{X}$ and $\mathbf{G}$ which locates at a half of the conoid height. By direct calculations,

$$
\left(x^{2}-z^{2}\right) y-(\mu-\Gamma) x z=0,
$$

which is the algebraic equation for time-like Plücker conoid. The time-like Plücker conoid given by Equation (32) which has two structure functions of the first order and it depends only on their difference; $\mu-\Gamma=1,-0.9 \leq \psi \leq 0.9,-1.5 \leq v$ $\leq 1.5$ (Figure 3 ). Furthermore, one can get a second-order equation in $x / z$ in which its solutions are given by

$$
\frac{x}{z}=\frac{1}{2 y}\left[\mu-\Gamma \pm \sqrt{(\mu-\Gamma)^{2}-4 y^{2}}\right] .
$$

By equating the discriminant of Equation (33) to zero, we get the limits of the time-like Plücker conoid. Hence, the two extreme positions are given by

$$
y= \pm \frac{\mu-\Gamma}{2},
$$

which gives the positions of the two torsal planes $\pi_{1}, \pi_{2}$, and each one of them contains one torsal line $L$. Further, $h(u)$ in Equation (27) is not a periodic function and has at most two extreme values, the structure functions $\Gamma$ and $\mu$. Therefore, the oriented lines $\mathbf{X}$ and $\mathbf{G}$ are principal axes of the timelike Plücker conoid. However, the geometric aspects are discussed as follows:

(1) If $h(t) \neq 0$, then there are two real torsal lines $L_{1}$ and $L_{2}$ passing through the point $(0, y, 0)$ only if $y<(\mu$ $-\Gamma) / 2$; for the two limit points $y= \pm(\mu-\Gamma) / 2$, they coincide with the principal axes $\mathbf{X}$ and $\mathbf{G}$

(2) If $h(t)=0$, then the two torsal lines $L_{1}$ and $L_{2}$ are represented by

$$
\frac{x}{z}:=\operatorname{coth} \psi= \pm \sqrt{\frac{\mu}{\Gamma}}, y= \pm \sqrt{\Gamma \mu}
$$

Equation (35) means that the two torsal lines $L_{1}$ and $L_{2}$ are orthogonal each other in the Lorentzian sense. Furthermore, transition from polar coordinates to Cartesian coordinates could be completed by substituting

$$
x=\frac{\cosh \psi}{\sqrt{h}}, z=\frac{\sinh \psi}{\sqrt{h}},
$$

into Hamilton's formula; one obtains the equation

$$
D:|\Gamma| x^{2}-|\mu| z^{2}=1,
$$

of a conic section. This conic section is a Minkowski version of the Dupin indicatrix of surfaces theory in Euclidean 3space.

3.1.1. Serret-Frenet Motion. If $(X)$ is a time-like developable ruled surface, that is, $\mu=0$, in this case, Dupin's indicatrix is a set of parallel lines represented by $x^{2}=|1 / \Gamma|$, and the Blaschke frame $\{\mathbf{x}, \mathbf{t}, \mathbf{g}\}$ coincides with the classical Serret-Frenet frame, and then, the striction curve $\mathbf{C}$ becomes the edge of regression of $(X)$. Hence, $p$ and $q$ are the curvature $\kappa$ and the torsion $\tau$ of $\mathbf{C}$, respectively. Moreover, $q^{*}=1$ and $\Gamma \tau=$ 1 ( $\Gamma$ is the radius of torsion of $\mathbf{C}$ ). By similar argument, we can also have the following equations:

$h(t)=\frac{1}{\tau} \cosh ^{2} \psi=\frac{\tau}{\tau^{2}-\kappa^{2}}, \psi^{*}(t)=-\frac{1}{2} \Gamma \sinh 2 \psi=-\frac{\kappa}{\tau^{2}-\kappa^{2}}$.

The corresponding time-like Plücker conoid is

$$
M: \mathbf{y}(t, v)=\left(0, \psi^{*}, 0\right)+v(\cosh \psi, 0, \sinh \psi), \quad v \in \mathbb{R} \text {. }
$$

This yields

$$
\left(x^{2}-z^{2}\right) y+\Gamma x z=0 .
$$

3.2. Time-like Ruled Surfaces with Constant Disteli-Axis. A time-like ruled surface $(X)$ is defined as a constant Disteliaxis ruled surface if the dual angle between the ruling of 
$(X)$ and the Disteli-axis is always constant. Thus, when we say $(X)$ is a time-like constant Disteli-axis, we mean that all the rulings of $(X)$ have a constant Lorentzian dual angle from its Disteli-axis.

The dual arc length $d \widehat{s}=d s+\varepsilon d s^{*}$ of $\mathbf{X}(t) \in \mathbb{H}_{+}^{2}$ is

$$
\widehat{s}(t)=\int_{0}^{t} P d t=\int_{0}^{t} p(1+\varepsilon \mu) d t .
$$

After that, we will use the dual arc length parameter $\widehat{s}$ instead of $t$. If the prime means to differentiation as $\widehat{s}$, then from Equation (15), we get

$$
\left(\begin{array}{c}
\mathbf{X}^{\prime} \\
\mathbf{T}^{\prime} \\
\mathbf{G}^{\prime}
\end{array}\right)=\left(\begin{array}{ccc}
0 & 1 & 0 \\
1 & 0 & \Sigma \\
0 & -\Sigma & 0
\end{array}\right)\left(\begin{array}{c}
\mathbf{X} \\
\mathbf{T} \\
\mathbf{G}
\end{array}\right)=\Omega \times\left(\begin{array}{c}
\mathbf{X} \\
\mathbf{T} \\
\mathbf{G}
\end{array}\right),
$$

where $\Omega=\Sigma \mathbf{X}+\mathbf{G}$. Thus, we may write the following relationships:

$$
\begin{aligned}
\widehat{\kappa}(\widehat{s}) & :=\kappa+\varepsilon \kappa^{*}=\sqrt{\Sigma^{2}-1}=\frac{1}{\sinh \psi}=\frac{1}{\tilde{\rho}}, \widehat{\tau}(\widehat{s}):=\tau+\varepsilon \tau^{*} \\
& = \pm \frac{\Sigma^{\prime}}{\Sigma^{2}-1}= \pm \Psi^{\prime}
\end{aligned}
$$

where $\widehat{\kappa}$ and $\widehat{\tau}$ are the dual curvature function and the dual torsion function of $\mathbf{X}(\widehat{s}) \in \mathbb{H}_{+}^{2}$, respectively. The terms found in Equation (43) are such as to their counterparts in 3dimensional hyperbolic spherical geometry.

Definition 4. For a one-parameter hyperbolic dual motion, at an instant $\widehat{s} \in \mathbb{D}$, an oriented time-like line $Z$ in fixed space will be said to be time-like $\mathbf{B}_{k}$-Disteli-axis of $(X)$ if for all $i$ such that $1 \leq i \leq k,<\boldsymbol{Z}, \mathbf{X}^{i}(\widehat{s})>=0$, but $\left\langle\boldsymbol{Z}, \mathbf{X}^{k+1}(\widehat{s})>\neq 0\right.$. Here, $\mathbf{X}^{i}$ denotes the $i$-th derivatives of $\mathbf{X}$.

Via this definition, consider the Lorentzian dual angle

$$
\tilde{\rho}=\cosh ^{-1}(<\mathbf{Z}, \mathbf{X}>)
$$

such that $\mathbf{X}$ and $\mathbf{Z}$ have the same time orientation and $\mathbf{Z}$ and $\tilde{\rho}$ stay fixed up to the second order at $\widehat{s}=\widehat{s}_{0}$, i.e.,

$$
\begin{gathered}
\tilde{\rho}^{\prime}\left|\widehat{s}=\widehat{s}_{0}=0, \mathbf{X}^{\prime}\right| \widehat{s}=\widehat{s}_{0}=\mathbf{0}, \\
\tilde{\rho}^{\prime \prime}\left|\widehat{s}=\widehat{s}_{0}=0, \mathbf{X}^{\prime \prime}\right| \widehat{s}=\widehat{s}_{0}=\mathbf{0} .
\end{gathered}
$$

We have for the first order

$$
<\mathbf{X}^{\prime}, \mathbf{Z}>=0
$$

and for the second-order properties

$$
<\mathbf{X}^{\prime \prime}, \mathbf{Z}>\mid=0 \text {. }
$$

Then, $\tilde{\rho}$ will be invariant in the second approximation if and only if $\boldsymbol{Z}$ is the time-like Disteli-axis $\mathbf{B}$ of $(X)$, that is,

$$
\tilde{\rho}^{\prime}=\tilde{\rho}^{\prime \prime}=0 \Leftrightarrow \mathbf{Z}=\frac{\mathbf{X}^{\prime} \times \mathbf{X}^{\prime \prime}}{\left\|\mathbf{X}^{\prime} \times \mathbf{X}^{\prime \prime}\right\|}= \pm \mathrm{B} .
$$

By the definition of the time-like Disteli-axis, we have the dual frame

$$
\mathbf{U}_{1}=\mathbf{B}(\widehat{s}), \mathbf{U}_{2}(\widehat{s})=\frac{\mathbf{B}^{\prime}}{\left\|\mathbf{B}^{\prime}\right\|}, \mathbf{U}_{3}(\widehat{\boldsymbol{s}})=\mathbf{B} \times \mathbf{U}_{2},
$$

as the Blaschke frame along $\mathbf{B}$. Thus, the calculations give that

$$
\left(\begin{array}{c}
\mathbf{U}_{1} \\
\mathbf{U}_{2} \\
\mathbf{U}_{3}
\end{array}\right)=\left(\begin{array}{ccc}
\cosh \Psi & 0 & \sinh \Psi \\
\sinh \Psi & 0 & \cosh \Psi \\
0 & -1 & 0
\end{array}\right)\left(\begin{array}{l}
\mathbf{X} \\
\mathbf{T} \\
\mathbf{G}
\end{array}\right)
$$

The variations of this frame are analogous to Equation (15) and are given by

$$
\left(\begin{array}{c}
\mathbf{U}_{1}^{\prime} \\
\mathbf{U}_{2}^{\prime} \\
\mathbf{U}_{3}^{\prime}
\end{array}\right)=\left(\begin{array}{ccc}
0 & \Psi^{\prime} & 0 \\
\Psi^{\prime} & 0 & \widehat{\kappa} \\
0 & -\widehat{\kappa} & 0
\end{array}\right)\left(\begin{array}{c}
\mathbf{U}_{1} \\
\mathbf{U}_{2} \\
\mathbf{U}_{3}
\end{array}\right)=\tilde{\Omega} \times\left(\begin{array}{c}
\mathbf{U}_{1} \\
\mathbf{U}_{2} \\
\mathbf{U}_{3}
\end{array}\right),
$$

where $\tilde{\Omega}(\widehat{s})=\widehat{\kappa} \mathbf{U}_{1}+\Psi^{\prime} \mathbf{U}_{3}$. Comparing Equation (42) with Equation (51), we have that the relative dual velocity is

$$
\Omega-\tilde{\Omega}=\Psi^{\prime} \mathbf{T} .
$$

This shows that the Blaschke frame involves a further rotation around the central tangent $\mathbf{T}$, whose speed equals the dual torsion $\widehat{\tau}(s)$. Hence, we obtain that if $\widehat{\tau}(\widehat{s})=\tau+\varepsilon$ $\tau^{*}=0\left(\Sigma^{\prime}(t)=\gamma^{\prime}(t)+\varepsilon(\Gamma(t)-\mu(t) \gamma(t))^{\prime}=0\right)$, that is, $\psi$ and $\psi^{*}$ are constants, then the time-like Disteli-axis is fixed up to the second order and the time-like line $\mathbf{X}$ moves on it with constant pitch $h$. Thus kinematically, the time-like ruled surface $(X)$ is traced by a hyperbolic one-parameter screw motion of constant pitch $h$ along the constant timelike Disteli-axis $\mathbf{B}$, by the time-like line $\mathbf{X}$ at a constant Lorentzian distance $\psi^{*}$ and constant Lorentzian angle $\psi$ relative to $\mathbf{B}$. Hence, we have the following.

Theorem 5. $(X)$ is a time-like ruled surface with constant Disteli-axis if and only if (a) $\gamma=$ constant and (b) $\Gamma-\mu \gamma=$ constant.

Now, we construct the time-like ruled surfaces for which the Disteli-axis is constant. Thus, from Equation (42), we have the following ordinary differential equation:

$$
\mathbf{X}^{\prime \prime \prime}+\tilde{\kappa}^{2} \mathbf{X}^{\prime}=\mathbf{0}
$$


Then, without loss of generality, we may assume $\mathbf{X}^{\prime}(0)$ $=(0,1,0)$. Under such initial condition, a space-like dual unit vector $\mathbf{X}^{\prime}$ is given by

$\mathbf{X}^{\prime}(\widehat{s})=A_{1} \sin (\tilde{\mathcal{k}} \widehat{s}) \mathbf{F}_{1}+\left(\cos (\tilde{\mathcal{K}} \widehat{s})+A_{2} \sin (\tilde{\mathcal{K}} \widehat{s})\right) \mathbf{F}_{2}+A_{3} \sin (\tilde{\mathcal{K}} \widehat{s}) \mathbf{F}_{3}$,

where $A_{1}, A_{2}$, and $A_{3}$ are some dual constants satisfying $A_{1}^{2}$ $-A_{3}^{2}=1$ and $A_{2}=0$. From this, we can obtain

$$
\begin{aligned}
\mathbf{X}(\widehat{\mathcal{s}})= & \left(-\tilde{\rho} A_{1} \cos (\tilde{\mathcal{\kappa}} \widehat{\mathcal{S}})+D_{1}\right) \mathbf{F}_{1}+\tilde{\rho} \sin (\tilde{\mathcal{\kappa}} \widehat{s}) \mathbf{F}_{2} \\
& +\left(-\tilde{\rho} A_{3} \cos (\tilde{\mathcal{\kappa}} \widehat{\mathcal{S}})+D_{3}\right) \mathbf{F}_{3},
\end{aligned}
$$

where $D_{1}, D_{3}$ are some dual constants satisfying $A_{3} D_{3}-A_{1}$ $D_{1}=0$ and $D_{3}^{2}-D_{1}^{2}=\tilde{\rho}^{2}+1$. If we adopt the dual coordinate transformation such that

$$
\left(\begin{array}{c}
\bar{X}_{1} \\
\bar{X}_{2} \\
\bar{X}_{3}
\end{array}\right)=\left(\begin{array}{ccc}
A_{1} & 0 & -A_{3} \\
0 & 1 & 0 \\
-A_{3} & 0 & A_{1}
\end{array}\right)\left(\begin{array}{l}
X_{1} \\
X_{2} \\
X_{3}
\end{array}\right)
$$

with respect to the new coordinates $\bar{X}_{i}$, the dual unit vector $\mathbf{X}(\widehat{\mathbf{s}})$ becomes

$$
\mathbf{X}(\widehat{s})=\tilde{\rho} \cos (\tilde{\mathcal{K}} \widehat{s}) \mathbf{F}_{1}+\tilde{\rho} \sin (\tilde{\mathcal{K}} \widehat{s}) \mathbf{F}_{2}+D \mathbf{F}_{3},
$$

for a constant dual $D=A_{1} D_{3}-A_{3} D_{1}$, with $D=\mp \cosh \Psi$. It is noted that $\mathbf{X}(\widehat{s})$ does not depend on the choice of the lower sign or upper sign of $\mp$. Therefore, through the paper, we choose the upper sign, that is,

$$
\mathbf{X}(\Theta)=\sinh \Psi \cos (\widetilde{\mathcal{K}} \widehat{s}) \mathbf{F}_{1}+\sinh \Psi \sin (\widetilde{\mathcal{K}} \widehat{s}) \mathbf{F}_{2}-\cosh \Psi \mathbf{F}_{3},
$$

where $\Theta=\vartheta+\varepsilon \vartheta^{*}=\tilde{\kappa} \widehat{s}$. This means that the time-like lines $\mathbf{B}$ and $\mathbf{F}_{3}$ are coincident, and

$$
\psi=f_{1} \text { (real const.), } \psi^{*}=f_{2} \text { (real const.). }
$$

Since $\vartheta$ and $\vartheta^{*}$ are two-independent parameters, we can say that $(X)$ is, in general, a time-like line congruence in $\mathbb{L}_{f}$ -space. However, by separating the real and dual parts of Equation (58), respectively, we have

$$
\mathbf{x}\left(\vartheta, \vartheta^{*}\right)=(\sinh \psi \cos \vartheta, \sinh \psi \sin \vartheta,-\cosh \psi),
$$

$$
\mathbf{x}^{*}\left(\vartheta, \vartheta^{*}\right)=\left(\begin{array}{c}
x_{1}^{*} \\
x_{2}^{*} \\
x_{3}^{*}
\end{array}\right)=\left(\begin{array}{c}
\psi^{*} \cos \vartheta \cosh \psi-\vartheta^{*} \sin \vartheta \sinh \psi \\
\psi^{*} \sin \vartheta \cosh \psi+\vartheta^{*} \cos \vartheta \sinh \psi \\
-\psi^{*} \sinh \psi
\end{array}\right)
$$

Let $\boldsymbol{\alpha}\left(\alpha_{1}, \alpha_{2}, \alpha_{3}\right)$ denote a point on $\mathbf{X}$. Since $\boldsymbol{\alpha} \times \mathbf{x}=\mathbf{x}^{*}$, we have the system of linear equations in $\alpha_{1}, \alpha_{2}$, and $\alpha_{3}$ :

$$
\left.\begin{array}{l}
-\alpha_{3} \sin \vartheta \sinh \psi-\alpha_{2} \cosh \psi=x_{1}^{*}, \\
\alpha_{3} \cos \vartheta \sinh \psi+\alpha_{1} \cosh \psi=x_{2}^{*}, \\
-\alpha_{1} \sin \vartheta \sinh \psi+\alpha_{2} \cos \vartheta \sinh \psi=x_{3}^{*} .
\end{array}\right\}
$$

The matrix of coefficients of unknowns $\alpha_{1}, \alpha_{2}$, and $\alpha_{3}$ is

$$
\left(\begin{array}{ccc}
0 & -\cosh \psi & -\sin \vartheta \sinh \psi \\
\cosh \psi & 0 & \cos \vartheta \sinh \psi \\
-\sin \vartheta \sinh \psi & \cos \vartheta \sinh \psi & 0
\end{array}\right) \text {, }
$$

and therefore, its rank is 2 with $\psi \neq 0$ and $\vartheta \neq 0$. In addition, the rank of the augmented matrix

$$
\left(\begin{array}{cccc}
0 & \cosh \psi & -\sin \vartheta \sinh \psi & x_{1}^{*} \\
-\cosh \psi & 0 & \cos \vartheta \sinh \psi & x_{2}^{*} \\
-\sin \vartheta \sinh \psi & \cos \vartheta \sinh \psi & 0 & x_{3}^{*}
\end{array}\right)
$$

is 2 . Hence, this system has infinitely many solutions represented with

$$
\begin{gathered}
c \alpha_{1}=\psi^{*} \sin \vartheta+\left(\vartheta^{*}-\alpha_{3}\right) \tanh \psi \cos \vartheta, \\
\alpha_{2}=-\psi^{*} \cos \vartheta+\left(\vartheta^{*}-\alpha_{3}\right) \tanh \psi \sin \vartheta, \\
-\alpha_{1} \sin \vartheta \sinh \psi+\alpha_{2} \cos \vartheta \sinh \psi=x_{3}^{*} .
\end{gathered}
$$

Since $\alpha_{3}$ is taken at random, then we may take $\vartheta^{*}-\alpha_{3}$ $=0$. In this case, Equation (65) reduces to

$$
\alpha_{1}(\vartheta)=\psi^{*} \sin \vartheta, \alpha_{2}(\vartheta)=-\psi^{*} \cos \vartheta, \alpha_{3}(\vartheta)=-\vartheta^{*} .
$$

Thus, the director surface of this time-like line congruence is given by

$$
\alpha\left(\vartheta, \vartheta^{*}\right)=\left(\psi^{*} \sin \vartheta,-\psi^{*} \cos \vartheta,-\vartheta^{*}\right) .
$$

Let $\mathbf{m}\left(m_{1}, m_{2}, m_{3}\right)$ denote a point on this time-like line congruence. Hence, we obtain

$$
\mathbf{m}\left(\vartheta, \vartheta^{*}, v\right)=\alpha\left(\vartheta, \vartheta^{*}\right)+v \mathbf{x}\left(\vartheta, \vartheta^{*}\right), \quad v \in \mathbb{R},
$$

which consists of a family of time-like ruled surfaces $\mathbf{m}(\vartheta$, $\left.\vartheta_{0}^{*}, v\right), \mathbf{m}\left(\vartheta_{0}, \vartheta^{*}, v\right)$, and $\mathbf{m}\left(\vartheta(t), \vartheta^{*}(t), v\right)$. Here, $\vartheta_{0}^{*}, \vartheta_{0}$, and $t$ are real constants. By means of Equations (60), (68), and (70), we simply find that

$$
\left.\begin{array}{l}
c m_{1}=\psi^{*} \sin \vartheta+v \sinh \psi \cos \vartheta, \\
m_{2}=-\psi^{*} \cos \vartheta+v \sinh \psi \sin \vartheta, \\
m_{3}=-\vartheta^{*}-v \cosh \psi,
\end{array}\right\}
$$

or by eliminating $\vartheta$, we have 


$$
(X): \frac{m_{1}^{2}}{\psi^{* 2}}+\frac{m_{2}^{2}}{\psi^{* 2}}-\frac{M_{3}^{2}}{n^{2}}=1,
$$

where $n=\psi^{*} \operatorname{coth} \psi$ and $M_{3}=m_{3}+9^{*}$. Then, $(X)$ is twoparameter Lorentzian spheres. The intersection of each Lorentzian sphere and the corresponding space-like plane $M_{3}$ $:=m_{3}+\vartheta^{*}=0$ is $m_{1}^{2}+m_{2}^{2}=\psi^{* 2}$. Therefore the envelope of $(X)$ is the time-like cylinder $m_{1}^{2}+m_{2}^{2}=\psi^{* 2}$. Notice that if $\vartheta^{*}=0$, then

$$
(X): \frac{m_{1}^{2}}{\psi^{* 2}}+\frac{m_{2}^{2}}{\psi^{* 2}}-\frac{m_{3}^{2}}{n^{2}}=1 .
$$

3.2.1. Constant Parameter Time-like Ruled Surfaces. A relation such as $F\left(\vartheta, \vartheta^{*}\right)=0$, between the parameters, restricts Equation (58) (resp. (70)) to a one-parameter set of timelike lines, that is, a time-like ruled surface in the congruence. Therefore, if we select $\vartheta^{*}=h \vartheta, h$ indicating the pitch of the motion $\mathbb{H}_{+m}^{2} / \mathbb{H}_{+f}^{2}$, and $\vartheta$ as the motion parameter, then Equation (58) (resp. (70)) performs a time-like ruled surface in $\mathbb{L}_{f}$-space. Thus,

$$
\left(\begin{array}{l}
\mathbf{X} \\
\mathbf{T} \\
\mathbf{G}
\end{array}\right)=\left(\begin{array}{ccc}
\sinh \Psi \cos \Theta & \sinh \Psi \sin \Theta & -\cosh \Psi \\
-\sin \Theta & \cos \Theta & 0 \\
\cosh \Psi \cos \Theta & \cosh \Psi \sin \Theta & -\sinh \Psi
\end{array}\right)\left(\begin{array}{l}
\mathbf{F}_{1} \\
\mathbf{F}_{2} \\
\mathbf{F}_{3}
\end{array}\right)
$$

In this case, we get

$$
\begin{aligned}
\frac{d}{d \vartheta}\left(\begin{array}{l}
\mathbf{X} \\
\mathbf{T} \\
\mathbf{G}
\end{array}\right)= & \left(\begin{array}{ccc}
0 & (1+\varepsilon h) \sinh \Psi & 0 \\
(1+\varepsilon h) \sinh \Psi & 0 & (1+\varepsilon h) \cosh \Psi \\
0 & -(1+\varepsilon h) \cosh \Psi & 0
\end{array}\right) \\
& \cdot\left(\begin{array}{l}
\mathbf{X} \\
\mathbf{T} \\
\mathbf{G}
\end{array}\right) .
\end{aligned}
$$

Thereby, the Blaschke invariants are

$$
P=(1+\varepsilon h) \sinh \Psi, Q=(1+\varepsilon h) \cosh \Psi, \operatorname{coth} \Psi=\frac{Q}{P} .
$$

From the real and dual parts of the last equations, we obtain

$$
\mu=h+\psi^{*} \operatorname{coth} \psi, \Gamma=h+\psi^{*} \tanh \psi, \gamma=\operatorname{coth} \psi,
$$

where $\mu, \Gamma$, and $\gamma$ are constants. Hence, $(X)$ is a constant parameter time-like ruled surface. We now simply find the base curve as

$$
\alpha(\vartheta)=\left(\psi^{*} \sin \vartheta,-\psi^{*} \cos \vartheta,-h \vartheta\right) .
$$

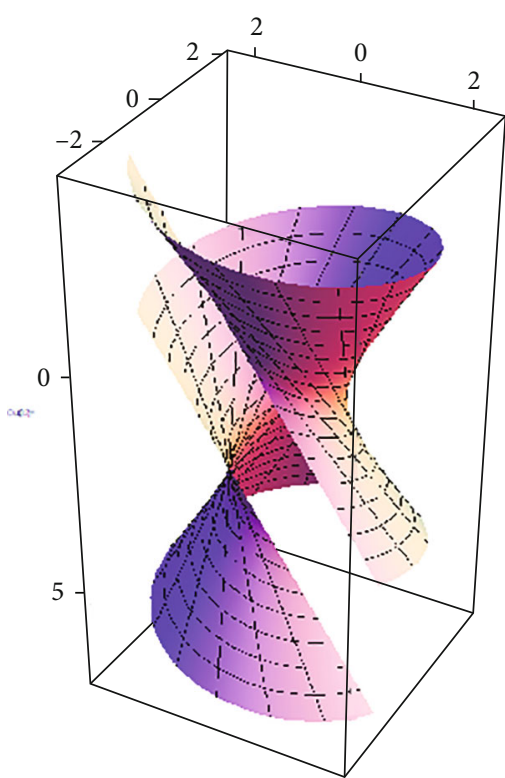

Figure 4: $(X)$ with $h=1$ and $\psi^{*}=0.5$.

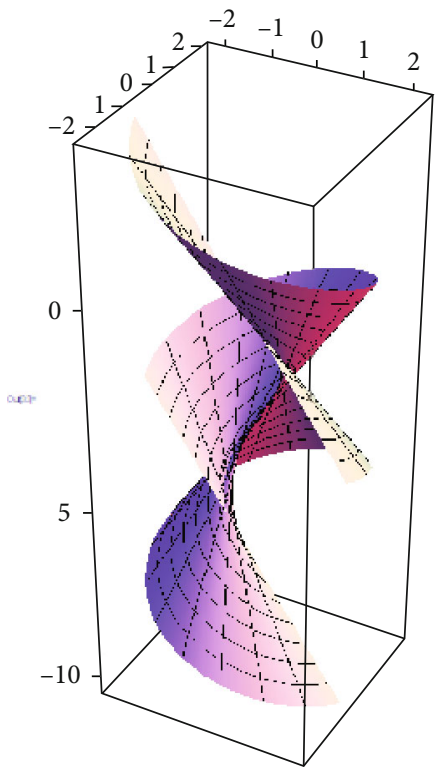

Figure 5: $(X)$ with $h=0.5$ and $\psi^{*}=1$.

It can be shown that $\left\langle\alpha^{\prime}, \mathbf{x}^{\prime}\right\rangle=0$, so the base curve of $(X)$ is its striction curve. Also, it can be show that $\alpha(\vartheta)$ is a space-like (resp., a time-like) if and only if $\left|\psi^{*}\right|>|h|$ (resp., $\left.\left|\psi^{*}\right|<|h|\right)$. For the curvature $\kappa$, and the torsion $\tau$, we can find the following calculations simply

$$
\kappa(\vartheta)=\frac{\psi^{*}}{\psi^{* 2}-h^{2}}, \tau(\vartheta)=\frac{h}{\psi^{* 2}-h^{2}} .
$$

Hence, $\alpha(\vartheta)$ is a space-like (resp., a time-like) helix if and only if $\left|\psi^{*}\right|>|h|$ (resp. $\left.\left|\psi^{*}\right|<|h|\right)$. Furthermore, we have 


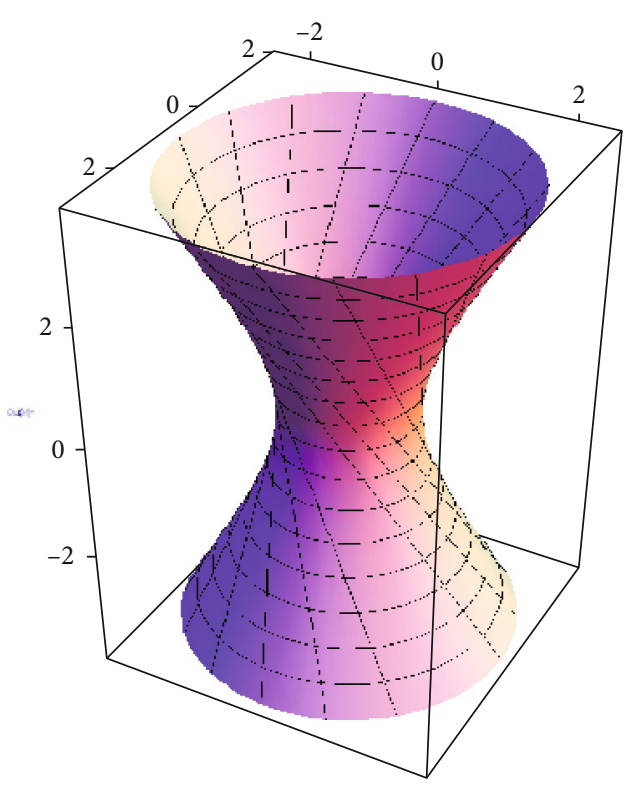

FIGURE 6: $(X)$ with $h=0$ and $\psi^{*}=1$.

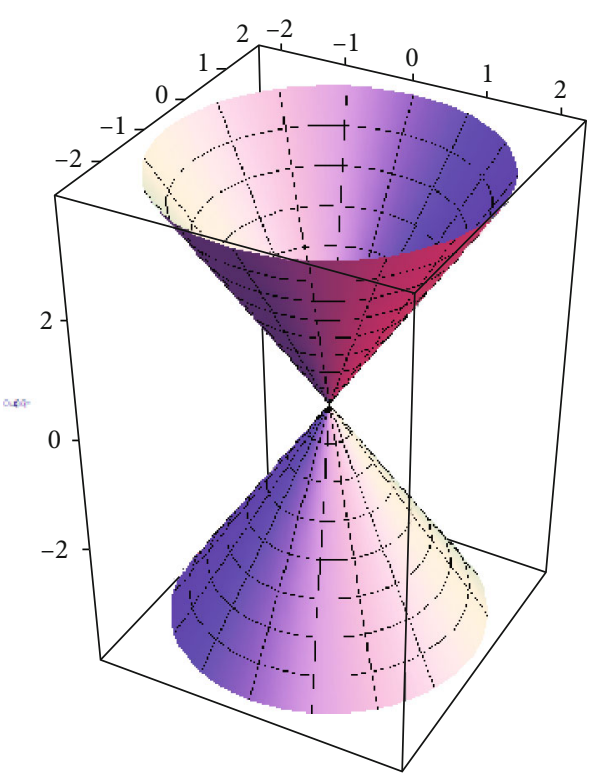

Figure 7: $(X)$ with $h=0=\psi^{*}=0$.

$(X): \mathbf{m}(\vartheta, v)=\left(\psi^{*} \sin \vartheta+v c_{1} \cos \vartheta,-\psi^{*} \cos \vartheta+v c_{1} \sin \vartheta,-h \vartheta-c_{2} v\right)$

where $c_{1}=\sinh \psi, c_{2}=\cosh \psi, \psi=0.7, \vartheta \in[0,2 \pi]$, and $v \in$ $[-4,4]$. According to Equation (80), we have the following:

(1) Time-like general helicoids: for $h=0.5$ (resp., $h=1$ ) and $\psi^{*}=1$ (resp., $\psi^{*}=0.5$ ), the graph of the surfaces are shown in Figures 4 and 5, respectively

(2) Lorentzian sphere: for $h=0, \psi^{*}=1$, the graph of the surface is shown in Figure 6

(3) Time-like cone: Figure 7 shows the surface $(X)$ with $\psi^{*}=h=0$

\section{Conclusion}

In this work, we analyze a certain class of time-like ruled with constant Disteli-axis in Minkowski 3-space $\mathbb{E}_{1}^{3}$. As a result, the time-like ruled surface generated by a time-like line undergoing a Lorentzian screw motion is examined in detail. We hope that the study of spatial kinematics in Minkowski 3-space $\mathbb{E}^{3}$ via line geometry may shed some light on present research problems and probably propose new ones.

\section{Data Availability}

No data were used to support this study.

\section{Conflicts of Interest}

The authors declare that they have no conflicts of interest.

\section{References}

[1] O. Bottema and B. Roth, Theoretical Kinematics, North-Holland Press, New York, 1979.

[2] A. Karger and J. Novak, Space Kinematics and Lie Groups, Gordon and Breach Science Publishers, New York, 1985.

[3] H. Pottman and J. Wallner, Computational Line Geometry, Springer-Verlag, Berlin, Heidelberg, 2001.

[4] R. A. Abdel-Baky and F. R. Al-Solamy, "A new geometrical approach to one-parameter spatial motion," Journal of Engineering Mathematics, vol. 60, no. 2, pp. 149-172, 2008.

[5] R. A. Abdel-Baky and R. A. Al-Ghefari, "On the oneparameter dual spherical motions," Computer Aided Geometric Design, vol. 28, no. 1, pp. 23-37, 2011.

[6] R. A. Al-Ghefari and R. A. Abdel-Baky, "Kinematic geometry of a line trajectory in spatial motion," Journal of Mechanical Science and Technology, vol. 29, no. 9, pp. 3597-3608, 2015.

[7] R. A. Abdel-Baky, "On the curvature theory of a line trajectory in spatial kinematics," Korean Mathematical Society, vol. 34, no. 1, pp. 333-349, 2019.

[8] M. Çimdiker Aslan and G. Aydın Şekerci, "Dual curves associated with the Bonnet ruled surfaces," International Journal of Geometric Methods in Modern Physic, vol. 17, no. 13, article 2050204, 2020.

[9] N. Alluhaibi, "Ruled surfaces with constant Disteli-axis," AIMS Mathematics, vol. 5, no. 6, pp. 7678-7694, 2020.

[10] N. Alluhaibi and R. A. Abdel-Baky, "On the one-parameter Lorentzian spatial motions," International Journal of Geometric Methods in Modern Physics, vol. 16, no. 12, article 1950197, 2019.

[11] N. Alluhaibi and R. A. Abdel-Baky, "Kinematic geometry of hyperbolic dual spherical motions and Euler-Savary's equation," International Journal of Geometric Methods in Modern Physics, vol. 17, no. 5, article 2050079, 2020.

[12] R. A. Abdel-Baky and Y. Unluturk, "A new construction of time-like ruled surfaces with constant Disteli-axis," Honam Mathematical Journal, vol. 42, no. 3, pp. 551-568, 2020.

[13] N. H. Abdel-All, R. A. Abdel-Baky, and F. Hamdoon, "Ruled surfaces with time-like rulings," Applied Mathematics and Computation, vol. 147, no. 1, pp. 241-253, 2004.

[14] R. Lopez, "Differential geometry of curves and surfaces in Lorentz-Minkowski space,” 2008, https://arxiv.org/abs/0810 .3351 . 
[15] B. O’Neil, Semi-Riemannian Geometry, with Applications to Relativity, Academic Press, New York, 1983.

[16] J. Walfare, Curves and surfaces in Minkowski space, [Ph.D. thesis], K.U. Leuven, Faculty of Science, Leuven, 1995.

[17] W. Kuhnel, Differential Geometry, American Mathematical Soc, 2nd edition, 2006. 